Mots. Les langages du politique

Guerres et paix. Débats, combats, polémiques

\title{
Entre décence et obscénité : ce qui se dit de la guerre dans Le temps retrouvé
}

Proust's appraisal of war in Time Regained: a mixture of decency and obscenity

Entre decencia y obscenidad: lo que se dice de la guerra en Le temps retrouvé de Proust

\section{Elisheva Rosen}

\section{OpenEdition}

\section{Journals}

Édition électronique

URL : https://journals.openedition.org/mots/2273

DOI : $10.4000 /$ mots. 2273

ISSN : 1960-6001

Éditeur

ENS Éditions

\section{Édition imprimée}

Date de publication : 1 novembre 2004

Pagination : 75-89

ISBN : 2-84788-064-X

ISSN : 0243-6450

\section{Référence électronique}

Elisheva Rosen, «Entre décence et obscénité : ce qui se dit de la querre dans Le temps retrouvé », Mots.

Les langages du politique [En ligne], 76 | 2004, mis en ligne le 21 avril 2008, consulté le 22 avril 2022.

URL : http://journals.openedition.org/mots/2273 ; DOI : https://doi.org/10.4000/mots.2273 


\section{Entre décence et obscénité : ce qui se dit de la guerre dans Le temps retrouvé}

La Recherche est intimement marquée du sceau de la Grande Guerre. L'œuvre était pratiquement terminée (du moins pour tout ce qui concerne sa conception) lorsque les évènements en ont interrompu la publication. Proust n'a cessé dès lors de remanier inlassablement son manuscrit, au risque de le vouer à cet inachèvement (relatif) qui caractérise désormais pour nous son texte, et dont les pages consacrées à la guerre portent, un peu plus que d'autres, l'empreinte ${ }^{2}$. Tout se passe comme si, sous la pression de l'actualité, Proust avait souhaité en quelque sorte mettre son œuvre, son esthétique et sa vision, à l'épreuve du réel. Revirement d'un écrivain qui jusque-là n'aurait accepté d'accueillir dans son texte les échos du monde que sous une forme assourdie ? Pas vraiment, puisque dans une telle éventualité, c'est une autre œuvre que Proust aurait dû entreprendre, plutôt que de remanier son texte. En prenant le parti de suralimenter la Recherche et notamment d'y inscrire la guerre, il semble bien plutôt que ce soit la pertinence (historique) de son œuvre qu'il ait choisi de mettre en relief, en accusant certains de ses traits, qui trop feutrés au vu du changement des circonstances, eussent pu, après-guerre, laisser croire à une indifférence d'esthète à l'évènement. Mais dans cette hypothèse, les pages consacrées à la guerre dans Le temps retrouvé n'en apparaissent que plus singulières. Nées de la conjoncture, elles s'y inscrivent si résolument en porte-à-faux, qu'elles ne laissent pas d'étonner. Étrange manière que d'accuser la pertinence d'une œuvre en écrivant ostensiblement, dans et à partir de l'actualité, à rebours de l'actualité. C'est à n'en pas douter un comble, l'un des plus fabuleux sans doute, que Proust ait pu envisager, lui

1. Université de TelAviv - erosen@post.tau.ac.il

2. Pour l'histoire du texte voir Marcel Proust, À la Recherche du temps perdu, Le temps retrouvé, édition réalisée sous la direction de J. Milly, édition du texte, introduction, bibliographie par B. Brun, 1986, Paris, Flammarion (GF). Toutes nos citations renvoient à cette édition. Quelques variantes de ponctuation proviennent de l'édition Gallimard (Blanche). Voir également la notice de J.-L. Rey et B. Rogers, 1989, dans $A$ la recherche du temps perdu, édition publiée sous la direction de J.-Y. Tadié, Paris, Gallimard (Pléiade), vol. IV, p. 1146-1175. 
qui, comme Barthes ${ }^{3}$ l'a suggéré, affectionnait ce jeu, sa charge ludique, ses potentialités esthétiques et heuristiques.

\section{Un texte insensé ?}

À quoi ressemble Le temps retrouvé si on l'envisage en regard du patriotisme exacerbé des années de la Grande Guerre ? Le long passage consacré à la guerre semble tout d'abord parfaitement futile. Il s'occupe de questions de mode féminine, de style et d'art, de conversations oiseuses dans les salons. Autant de considérations dont la pertinence en regard de l'agenda de l'heure est pour le moins énigmatique. Le futile, en temps de guerre, relève de l'insignifiant, quand il ne passe pas pour franchement indécent. Mais précisément, le texte proustien prévient ce type de dilemme... en l'aggravant. Indécent est un euphémisme pour le qualifier : il est aussi très manifestement obscène. Sodome occupe le devant de la scène et il en va un peu comme si la guerre n'intervenait que pour favoriser les amours homosexuelles en leur offrant, en dépit de la pénurie d'hommes, des occasions inespérées. Ainsi des couloirs du métro où l'on s'abrite lors des bombardements, et où les corps se rencontrent furtivement à la faveur de l'obscurité. Ainsi de cet hôtel très particulier de Jupien, où les permissionnaires s'évertuent à jouer tant bien que mal les apaches afin de satisfaire les plaisirs masochistes d'un baron de Charlus, et où les héros du front (Saint-Loup, en l'occurrence) oublient malencontreusement leur croix de guerre. Proust, à l'évidence, devance ses détracteurs éventuels en jouant de la surenchère. Comment ne pas crier au scandale devant pareille accumulation de scènes scabreuses?

Mais aussi pourquoi le faire ? Ce serait peut-être une réaction excessive face à des propos qui semblent surtout consternants, et qui pourraient bien émaner d'un esprit malade. Une hypothèse que le texte proustien confirme : le narrateur de la Recherche, pour tous les épisodes qui concernent la Grande Guerre, est quelqu'un qui fait de longs séjours dans une maison de santé : il en sort une première fois en aout 1914, puis définitivement en 1916, non parce qu'il est guéri, mais parce que faute de personnel, l'établissement a fermé ses portes. Proust devance en quelque sorte les réactions de ses lecteurs : il les conforte et en accrédite le bien-fondé. Il le fait d'ailleurs si bien qu'il les décontenance, car

3. R. Barthes, 1983, «Une idée de recherche», dans l'ouvrage collectif Recherche de Proust, Seuil (Points), p. 34-39. Pour d'autres exploitations du «comble» dans la Recherche, voir mon étude «Mondanités proustiennes : le tournant de Sodome et Gomorrhe» Marcel Proust 4, 2004, textes réunis par B. Brun et J. Hassine, Paris et Caen, Lettres modernes Minard, p. $235-248$. 
du même coup, c'est la raison d'être d'un tel récit qui ne laisse pas d'intriguer. S'il est insensé comme tout porte (un peu trop bien) à le croire, pourquoi nous le conter? Ainsi la figure du narrateur-malade prévient sans doute les objections très prévisibles du lecteur, mais ne l'en plonge pas moins dans la perplexité. Le dilemme s'aggrave du fait que la maladie du narrateur ne l'assimile pas pour autant à ces fous qui abondent dans la littérature et qui sont préposés à tenir un contre-discours, à se faire les porte-paroles d'un monde à l'envers. En somme, cette figure nous renvoie au problème des rapports qu'entretient le texte proustien avec le tout-venant des discours du moment.

\section{Un texte patriotique?}

Par rapport à ces discours, le texte proustien exhibe et assume sa déviance. Proust n'ignore pas les contraintes multiples et variées qui pèsent sur l'expression en temps de guerre. Il s'y montre au contraire extrêmement sensible. Mieux, il se targue à sa manière de s'y plier. Que le patriotisme soit de rigueur, il le reconnait volontiers, et il ne souhaite d'ailleurs nullement se soustraire à cette exigence. Il sait toutefois que son texte prête à équivoque, et que, c'est le moins que l'on puisse dire, le patriotisme n'est pas sa vertu la plus apparente ${ }^{4}$. C'est sans doute l'une des raisons qui l'incitent à faire du patriotisme l'un des leitmotive de son volume. Il en vient à multiplier les exemples qui visent, entre autre, à montrer que le patriotisme véritable n'est pas celui qui s'affiche. Ainsi de Saint-Loup qui se démène pour reprendre du service, mais qui n'en dit pas moins à qui veut bien l'entendre qu'il ne cherche pas à rejoindre les rangs de l'armée en faisant ouvertement état de sa peur. Bloch, au contraire, certain que sa myopie le protège d'une éventuelle mobilisation, est celui qui se fera une spécialité des tirades patriotiques jusqu'au jour où on lui annonce qu'il est apte au service. L'humour de cette double évocation donne lieu à des développements d'un ton plus sérieux. Si l'amour de la France reste tacite chez SaintLoup, c'est «par une espèce de délicatesse morale qui empêche d'exprimer les sentiments trop profonds et qu'on trouve tout naturels». Et le narrateur d'invoquer sa propre mère à l'appui :

Ma mère autrefois non seulement n'eût pas hésité une seconde à mourir pour ma grand'mère mais aurait horriblement souffert si on l'avait empêchée de le faire. Néanmoins il m'est impossible d'imaginer rétrospectivement dans sa bouche une phrase telle que : «Je donnerais ma vie pour ma mère». (p. 110)

4. J. Rancière, 1998, «Proust : la guerre, la vérité, le livre», dans La chair des mots. Politiques de l'écriture, Paris, Galilée, p. 137-153. Il n'y est toutefois que peu question de l'humour proustien et de sa fonction constructive. 
La délicatesse de Saint-Loup est d'ailleurs telle qu'il n'oublie pas de prendre des nouvelles de Françoise, se montrant soucieux de savoir si elle a réussi à faire réformer son neveu. Le narrateur visiblement apprécie cette délicatesse et la partage. Il note avec humour cette réplique de Françoise déclinant «sur un ton désespéré» l'offre d'une recommandation pour un général censé l'aider dans ses démarches : $[\ldots]$ «Oh ! non, ça ne servirait à rien, il n’y a rien à faire avec ce vieux bonhomme-là, c'est tout ce qu'il y a de pis, il est patriotique» [...] (p. 117). Incidemment, on se gardera bien de tenir ce propos, ou le comportement de Françoise, pour un indice de son absence foncière de patriotisme. Quelle leçon tirer de ces quelques exemples? La plus évidente se rapporte sans doute à l'auteur (et à son narrateur) : on est prié, en dépit des apparences, de ne pas douter de son patriotisme. Mais il y a plus, car ces exemples dénoncent plaisamment l'usage commun, policier et discriminatoire, qui se fait généralement du patriotisme. En nuançant l'usage du terme, Proust entend nous rendre sensible, par contraste, à la violence inhérente à la référence au patriotisme dans le langage courant en temps de guerre. Et l'on ne s'étonnera pas qu'il choississe de le faire, si l'on ose dire, en douceur. On aura noté au passage comment Proust se recommande des sentiments dans ces exemples, en se démarquant très manifestement du pathos patriotique en vigueur, un pathos qui (voir l'exemple de Bloch) doit s'entendre sur fond d'effroi. Ainsi ce n'est pas (uniquement) pour des raisons morales que Proust choisit de ne parler du (et de son) patriotisme autrement que sur ce mode détourné, c'est aussi et surtout pour des raisons à la fois esthétiques et politiques. L'art n'a pas à se plier, sans autre forme de procès, à des mots d'ordre, ce qui ne signifie pas pour autant qu'il doive les ignorer. C'est bien ce qui incite Proust à remettre en cause le bien fondé des injonctions d'un Barrès :

Dès le début de la guerre M. Barrès avait dit que l'artiste (en l'espèce Titien) doit avant tout servir la gloire de sa patrie. Mais il ne peut la servir qu'en étant artiste, c'est-à-dire qu'à condition, au moment où il étudie ces lois, institue ces expériences et fait ces découvertes aussi délicates que celles de la science, de ne pas penser à autre chose - fût-ce à la patrie - qu'à la vérité qui est devant lui. N'imitons pas les révolutionnaires qui par «civisme» méprisaient, s'ils ne les détruisaient pas, les œuvres de Watteau et de La Tour, peintres qui honorent davantage la France que tous ceux de la Révolution. (p. 281)

Proust a bien le sentiment qu'on puisse être tenté de rejeter son texte, si peu conforme aux attentes, par «civisme». Il plaide certes, là encore, pour sa chapelle. Mais ce qui est en cause, et la référence à la Révolution est choisie en conséquence, c'est aussi bien autre chose : il y va de l'histoire du roman moderne et de son rapport à la scène politique. Proust s'en prend de fait à la collusion de l'art et de la scène politique qui caractérise les époques de crise, et qui, il lui semble utile de le rappeler, n'est pas née de la guerre, même si celle-ci la 
réactive sous une forme nouvelle à l'enseigne du patriotisme. Il ne considère pas pour autant que l'art est dénué de pertinence politique. Encore moins, bien sûr, que la littérature n'engage pas la responsabilité civique de l'écrivain ${ }^{5}$. C'est bien au contraire parce qu'elle l'engage, que l'artiste doit veiller à l'autonomie de sa sphère d'action.

\section{Écrire dans la guerre, écrire la guerre}

Pour l'écrivain, tout le texte proustien le laisse entendre, la guerre est d'abord une grande leçon d'humilité. C'est sans doute quelque chose qui mérite d'être souligné. D'autant que c'est là aussi l'un des termes du différend qui l'oppose à Barrès, dont le programme implique pour l'écrivain la soumission à une servitude («servir la patrie») qui pourrait bien avoir pour contrepartie une forme difficilement tolérable d'arrogance. Ainsi, le choix proustien d'un narrateurmalade, figure oxymorique d'une (ir)responsabilité assumée, pour conter la guerre, relève sans doute pour une part d'un esprit de provocation. Il fait sens en regard de toutes les postures d'énonciation volontiers adoptées en temps de guerre, des postures qui sont autant de variantes sur le thème de l'individu chargé d'une mission, porteur d'un mandat. Pour Proust, si la guerre a bien une incidence sur l'artiste, ce n'est pas en tant qu'elle lui confère un nouveau mandat, ni une responsabilité nouvelle. C'est bien plutôt parce qu'elle le confronte à plus d'un titre à ses limites. Quelle que soit l'importance que Proust accorde à son activité (et la guerre le conforte aussi dans sa foi en la littérature, et en son propre génie), il ne s'agit jamais que d'une activité symbolique, qui ne peut, ni ne doit, soutenir la concurrence avec l'horreur des épreuves qu'inflige la guerre (ni d'ailleurs avec les souffrances qu'inflige la vie). S'il convient de garder, par décence, le sens des proportions, on ne saurait pour autant en tirer argument pour redéfinir, en fonction de la guerre, le rôle de l'écrivain. Certes, par rapport à l'esprit de 1914, une telle position se conçoit mal. Or là encore, Proust se montre particulièrement sensible à l'air du temps, aux limites qu'il impose à l'opinable. Il ne choisit pas par hasard de commencer son récit en 1916, et de n'évoquer 1914 que rétrospectivement et à distance. Tout son travail, très minutieux, se place sous le signe de la négociation avec le dicible de l'époque ${ }^{6}$ et avec ses contraintes. C'est de cet esprit de négociation que relèvent les consignes

5. Sur ce point, voir mon article «L'art du détour : la visée argumentative dans $\grave{A}$ la recherche du temps perdu» Littérature, $\mathrm{n}^{\circ} 119$, septembre 2000, p. 76-89.

6. Autant dire du discours social. Pour la définition de cette notion, voir M. Angenot, 1989, 1889. Un état du discours social, Montréal, Le Préambule. 
qu'il s'impose pour son roman dont celle, capitale, de lui imposer une restriction de champ. Il ne traitera pour l'essentiel que de la société civile, des gens de l'arrière (à Paris en règle générale, avec toutefois une brève incursion à Combray, un Combray ravagé par la guerre et qui se fait par le biais de lettres reçues de Gilberte). Pas question pour lui de parler du front et des combats, autrement qu'à partir de ce que peuvent en savoir ceux qui comme lui n'ont pas participé activement aux affrontements, et qui ne peuvent en avoir, au mieux, qu'une connaissance de seconde main. Ce qui ne l'empêche pas de témoigner sa compassion à l'égard des combattants, rencontrés lorsqu'ils arrivent en permission :

[...] c'était des rivages de la mort, vers lesquels ils allaient retourner, qu'ils venaient un instant parmi nous, incompréhensibles pour nous, nous remplissant de tendresse, d'effroi, et d'un sentiment de mystère, comme ces morts que nous évoquons, qui nous apparaissent une seconde, que nous n'osons pas interroger et qui du reste pourraient tout au plus nous répondre : «Vous ne pourriez pas vous figurer». Car il est extraordinaire à quel point, chez les rescapés du feu que sont les permissionnaires, chez les vivants ou les morts qu'un médium hypnotise ou évoque, le seul effet du contact avec le mystère soit d'accroître, s'il est possible, l'insignifiance des propos. (p. 128)

Autre notation du même genre dans cette vignette célèbre et émouvante :
À l'heure du dîner les restaurants étaient pleins; et si, passant dans la rue, je voyais un pauvre permissionnaire, échappé pour six jours au risque permanent de la mort, et prêt à repartir pour les tranchées, arrêter un instant ses yeux devant les vitres illu- minées, je souffrais comme à l'hôtel de Balbec quand des pêcheurs nous regardaient dîner, mais je souffrais davantage car je savais que la misère du soldat est plus grande que celle du pauvre, les réunissant toutes, et plus touchante encore parce qu'elle est plus résignée, plus noble, et que c'est d'un hochement de tête philosophe, sans haine, que prêt à repartir pour la guerre, il disait en voyant se bousculer les embusqués rete- nant leurs tables : «On ne dirait pas que c'est la guerre ici». (p. 102)

Ces brèves incursions dans cet autre monde du front, dans l'ailleurs des tranchées, se placent sous le signe de la limite que Proust s'interdit de franchir. C'est aussi que pour lui, la guerre n'est pas (voire ne saurait être) un sujet de roman (pas plus d'ailleurs que l'affaire Dreyfus, ou qu'à un autre titre, l'affaire Eulenbourg). Il n'ira pas jusqu'à dire ouvertement ce qu'il pense des romans de la guerre, dont le sien se démarque si manifestement. Son narrateur le laisse parfois entendre, allusivement, en prenant la remarque comme à son propre compte :

Car pour la même raison que de grands événements n'influent pas du dehors sur nos puissances d'esprit, et qu'un écrivain médiocre vivant dans une époque épique restera un tout aussi médiocre écrivain, ce qui était dangereux dans le monde c'était les dispositions mondaines qu'on y apporte. Mais par lui-même il n'était pas plus capable 
de vous rendre médiocre qu'une guerre héroïque de rendre sublime un mauvais poète. (p. 317)

[...] les héros d'un esprit médiocre et banal écrivant des poèmes au moment de leur convalescence se plaçaient pour décrire la guerre non au niveau des événements, qui en eux-mêmes ne sont rien, mais de la banale esthétique dont ils avaient suivi les règles jusque-là, parlant comme ils eussent fait dix ans plus tôt de la «sanglante aurore », du «vol frémissant de la victoire» etc., [...] (p. 124)

La guerre n'est pas un sujet de roman, mais il n'en reste pas moins inéluctable de lui faire une place dans le roman. Et s'il importe à Proust de s'en tenir pour cet épisode à la société civile, encore faut-il préciser que ce ne sera pas, comme on le dit souvent et un peu vite, pour écrire le roman des embusqués (auquel il est vrai, son ouvrage fait parfois penser) : à ce compte autant écrire un roman de la guerre. Parce que l'épisode de la guerre est indissociable d'une réflexion sur la littérature dont il se nourrit et qui le nourrit, Proust en viendra à produire un texte qui traite essentiellement de ce qui relève de sa sphère de compétence, le versant discursif de ce que les historiens nomment aujourd'hui la culture de guerre ${ }^{7}$.

\section{La culture de guerre}

$\mathrm{Au}$ fondement de la compréhension proustienne de la culture de guerre, il y a d'abord une certaine manière de concevoir le fossé qui sépare le monde de l'expérience des combattants, et celui d'une société civile en temps de guerre. Or ces deux mondes si différents ne peuvent pas être appréhendés en fonction des jeux d'oppositions si fréquemment invoqués - opposition des civils et des militaires, ou encore des combattants et des embusqués. Pour essentielles qu'elles soient, ces distinctions n'en atteignent pas moins un seuil où elles deviennent inopérantes. Ce seuil, l'écrivain y est d'autant plus sensible qu'il concerne l'ordre du discours. Or la scène discursive ${ }^{8}$, c'est une évidence mais il convient de la rappeler, reste pour l'essentiel, même en temps de guerre, et quelles que soient les mutations qu'elle puisse subir en la circonstance, celle de la société civile. Le soldat qui revient du front reste pratiquement muet («Vous ne pouvez pas vous figurer»), et ne peut s'intégrer à la communauté des «causeurs» qu'en

7. Voir notamment S. Audoin Rouzeau et A. Becker, 2000, 1418, retrouver la guerre, Paris, Gallimard. En mettant l'accent sur la dimension discursive, j'étends ici l'usage de la notion.

8. J'emploie scène discursive dans un sens qui ne recoupe pas la notion de scène d'énonciation utilisée en analyse du discours par D. Maingueneau. Voir P. Charaudeau et D. Maingueneau (dir.), 2002, Dictionnaire d'analyse du discours, Paris, Seuil, p. 515-518. 
disant des choses insignifiantes, convenues, ou renouant, avec à la rigueur une nuance, avec le style qui avait toujours été le sien. Nombre de remarques proustiennes mettent l'accent sur ce fait, constatant, à propos de Saint-Loup, que «le ton des entretiens était le même, la matière seule différait, et encore» (p. 128), pour préciser toutefois quelques pages plus loin :

Il faut dire pourtant que si la guerre n'avait pas grandi l'intelligence de Saint-Loup, cette intelligence $[\ldots]$ avait pris un brillant que je ne lui avais jamais vu. Quelle distance entre le jeune blondin qui jadis était courtisé par les femmes chic [...] et le discoureur, le doctrinaire qui ne cessait de jouer avec les mots! (p. 133)

Pour en revenir à la scène discursive, elle se voit placée, pour ainsi dire, sous le signe d'un double tropisme : celui d'une accommodation des civils à la guerre, celui d'une accommodation des combattants à cette scène métamorphosée. Ce sont ces tropismes, le jeu de leurs corrélations et l'examen de leurs effets qui sous-tendent la vision proustienne de la culture de guerre, et qui régissent le dispositif complexe de la mise en scène proustienne. Il importe tout d'abord de préciser que si ces deux grands tropismes que je viens d'évoquer présentent quelques affinités, et sont d'une certaine manière corrélatifs, ils ne sont pas symétriques pour autant. Il y a un sens de la différence que Proust entend maintenir dans son texte, un texte qui dans le même temps s'entend si bien à brouiller les différences, et qu'il y inscrira sur un double mode : celui des sites discursifs, celui du ton. La conversation de Saint-Loup qui, dans le roman, reste la figure majeure à partir de laquelle s'observe le tropisme du combattant (les autres ne sont évoquées que comme des silhouettes), ne s'entendra jamais, pour tout l'épisode de la guerre, dans les salons. Elle est placée sous le signe de l'amitié qui le lie au narrateur, qu'il rencontre dans la rue, auquel il vient rendre une visite rapide à l'occasion d'une permission, auquel il écrit pour lui envoyer des nouvelles du front. Incidemment, on notera que tout ce qui a trait aux questions d'art militaire, de stratégie, mais aussi plus concrètement (et plus rarement) de l'expérience du combattant est introduit par ce biais dans le roman. Pour autant, la conversation de Saint-Loup n'en reste pas moins un fragment de la conversation française à l'heure de la guerre, un fragment que Proust développe, qu'il traite avec déférence, mais qu'il accompagne aussi d'un commentaire critique qui le met en perspective. C'est dans une tout autre veine que Proust évoque à l'enseigne de la vie mondaine les ajustements de la société civile à la guerre. 


\section{À la guerre comme à la guerre}

Lorsque la comédie sociale s'ajuste à la guerre (une guerre qui entraine la promotion de $\mathrm{M}^{\text {mes }}$ Verdurin et Bontemps au rang de reines de Paris), ce n'est plus l'esprit qui règne dans les salons, c'est l'actualité :

$\mathrm{M}^{\mathrm{me}}$ Verdurin, pour communiquer les nouvelles, disait : «nous» en parlant de la France. «Hé bien voici : nous exigeons du roi de Grèce qu'il retire du Péloponnèse, etc.; nous lui envoyons, etc.» Et dans tous ses récits revenait tout le temps le GQG («j'ai téléphoné au GQG») abréviation qu'elle avait à prononcer le même plaisir qu'avaient naguère les femmes qui ne connaissaient pas le Prince d'Agrigente, à demander en souriant, quand on parlait de lui et pour montrer qu'elles étaient au courant : «Grigri ?», [...] (p. 96)

Le salon communique étroitement avec la presse, dans tous les sens d'ailleurs, comme le souligne $\mathrm{M}^{\mathrm{me}}$ Verdurin :

«C'est désolant, je vais téléphoner à Bontemps de faire le nécessaire pour demain, on a encore caviardé toute la fin de l'article de Norpois et simplement parce qu'il laissait entendre qu'on avait limogé Percin.» (p. 100)

Il ne s'agit pas pour Proust, dans cette collecte de tics de langage, de relever (ce qu'il fait aussi) que le snobisme est devenu guerrier, mais bien de montrer à l'œuvre le processus de mutation de la conversation française qui s'aligne plus que jamais sur le paradigme journalistique. Elle se nourrit de la presse, mais elle l'alimente également. L'exemple de Brichot, qui reprend du service en publiant des chroniques dans Le Temps l'atteste :

Ce fut ainsi que le journalisme (dans lequel Brichot se contentait en somme de donner tardivement, avec honneur et en échange d'émoluments superbes, ce qu'il avait gaspillé toute sa vie gratis et incognito dans le salon des Verdurin, car ses articles ne lui coûtaient pas plus de peine, tant il était disert et savant, que ses causeries) eût conduit $[\ldots]$ Brichot à une gloire incontestée... s'il n'y avait pas eu $\mathrm{M}^{\mathrm{me}}$ Verdurin. (p. 168)

Et le narrateur de commenter le style de ces articles, dont il apprécie l'érudition à l'encontre de $\mathrm{M}^{\mathrm{me}}$ Verdurin, qui la trouve ridicule :

Et à côté d'images qui ne voulaient rien dire du tout («les Allemands ne pourront plus regarder en face la statue de Beethoven; Schiller a dû frémir dans son tombeau; l'encre qui avait paraphé la neutralité de la Belgique était à peine séchée; Lénine parle, mais autant en emporte le vent de la steppe»), c'étaient des trivialités telles que : «Vingt mille prisonniers, c'est un chiffre. Notre commandement saura ouvrir l'œil et le bon. Nous voulons vaincre, un point c'est tout.» Mais, mêlé à tout cela, tant de savoir, tant d'intelligence, de si justes raisonnements ! (p. 169) 
Évoquer cette conversation française au moment de la guerre, c'est aussi pour Proust l'occasion de noter, façon Flaubert, sa niaiserie, et de montrer qu'elle procède d'un catalogue d'idées reçues et à recevoir. Lorsque $\mathrm{M}^{\mathrm{me}}$ Verdurin reproche à Brichot d'employer immodestement le je dans ses articles, et qu'il se met à substituer systématiquement un $o n^{9}$ à ce $j e$, on ne peut qu'apprécier l'ironie proustienne. La correction qui intervient dans le style de l'universitaire n'en met que mieux en relief le statut de son discours, son mode de production comme son mode d'emploi. C'est le conformisme belliqueux de l'expression, la prolifération des clichés et des lieux communs, les mots d'ordre qui caractérisent ce discours, mais aussi l'absence de mémoire. Les arguments varient avec une flagrante incohérence, que Proust note avec humour en reprenant à plusieurs reprises l'exemple de «la guerre sera courte», qui engendre, vu le démenti de l'évènement, des théories plus adaptées, proposées par ceux-là même qui avaient défendu cette thèse, mais ne s'en souviennent nullement. Évoquer la conversation française, c'est aussi pour Proust, réinscrire dans son texte les argumentaires de l'époque, dont il nous offre, chemin faisant, un échantillonnage varié et représentatif. Pourtant, il est important de noter qu'il ne considère pas cet ensemble de discours comme relevant du «bourrage de crâne» ou de la propagande. Aussi critique soit-il par rapport aux propos du temps, ce n'est pas du point de vue de Sirius qu'il se place en les rapportant, mais de celui de quelqu'un qui fait retour sur lui-même et envisage à distance ce qui a pu motiver, jusque dans les excès qu'il relève en les accentuant, l'adhésion à un tel régime discursif :

Le bourrage de crâne est un mot vide de sens. [...] Le véritable bourrage de crâne, on se le fait à soi-même par l'espérance, qui est une figure de l'instinct de conservation d'une nation, si l'on est vraiment membre vivant de cette nation. Pour rester aveugle sur ce qu'a d'injuste la cause de l'individu, Allemagne, pour reconnaître à tout instant ce qu'a de juste la cause de l'individu, France, le plus sûr n'était pas pour un Allemand de n'avoir pas de jugement, pour un Français d'en avoir, le plus sûr pour l'un ou pour l'autre était d'avoir du patriotisme. (p. 149)

Reste que le tropisme guerrier de la causerie, dont la presse est le pivot, se présente aussi pour tout ce qui pourrait se rapprocher de la pensée comme une chape de plomb étouffante. Les mots d'ordre et les contraintes sont telles que l'on n'y échappe que par des propos insignifiants, ou en leur payant un tribut franchement odieux. Ainsi de ce «brevet de civisme» dont doit se prémunir l'auteur d'un ouvrage sur Schiller :

9. Pour un commentaire stylistique de cet usage, voir A. Herschberg Pierrot, 2003, Stylistique de la prose, Paris, Éditions Belin, p. 28-29. 
À côté de cela certaines opinions artistiques, moins antigermaniques que pendant les premières années de la guerre, se donnaient cours pour rendre la respiration aux esprits étouffés, mais il fallait pour qu'on les osât présenter un brevet de civisme. Un professeur écrivait un livre remarquable sur Schiller et on en rendait compte dans les journaux. Mais avant de parler de l'auteur du livre, on inscrivait comme un permis d'imprimer qu'il avait été à la Marne, à Verdun, qu'il avait eu cinq citations, deux fils tués. Alors on louait la clarté, la profondeur de son ouvrage sur Schiller, qu'on pouvait qualifier de grand pourvu qu'on dît, au lieu de «ce grand Allemand», «ce grand Boche». C'était le mot d'ordre pour l'article et aussitôt on le laissait passer. (p. 223)

Intellectuellement calamiteux, comme l'indique cette dernière citation, les tropismes guerriers de la société civile, modèle Verdurin, sont également indissociables d'une forme de sensibilité pernicieuse. La guerre et le nouveau jeu de rôles auquel elle donne lieu, doublé d'une redistribution du personnel (la sociologie proustienne ${ }^{10}$ ), procède notamment pour ceux qui n'y participent pas directement de ce qui relève déjà d'une société du spectacle, comme l'indique cette célèbre vignette de $\mathrm{M}^{\mathrm{me}}$ Verdurin lisant son journal :

$\mathrm{M}^{\mathrm{me}}$ Verdurin, souffrant pour ses migraines de ne plus avoir de croissant à tremper dans son café au lait avait fini par obtenir de Cottard une ordonnance qui lui permit de s'en faire faire dans un certain restaurant [...]. Cela avait été presque aussi difficile à obtenir des pouvoirs publics que la nomination d'un général. Elle reprit son premier croissant le matin où les journaux narraient le naufrage du Lusitania. Tout en trempant le croissant dans le café au lait, et donnant des pichenettes à son journal pour qu'il pût se tenir grand ouvert sans qu'elle eût besoin de détourner son autre main des trempettes, elle disait : "Quelle horreur ! Cela dépasse en horreur les plus affreuses tragédies.» Mais la mort de tous ces noyés ne devait lui apparaître que réduite au milliardième, car tout en faisant, la bouche pleine, ces réflexions désolées, l'air qui surnageait sur sa figure, amené là probablement par la saveur du croissant, si précieux contre la migraine, était plutôt celui d'une douce satisfaction. (p. 148)

La guerre est aussi un film d'horreur dont on frémit douillettement, même si elle menace de frapper à votre porte. Cette vignette, par laquelle s'achève l'évocation des salons durant la guerre fait pendant au passage inaugural de l'épisode de la guerre qui traite des tropismes guerriers de la société civile en les plaçant sous le signe de la mode, et de son discours d'escorte. Le narrateur qui souhaite «entendre parler de la seule chose [qui l'intéresse,] la guerre», découvre en 1916 un Paris «qui faisait penser au Directoire», et où la parure féminine est «couleur du temps» :

10. Voir : C. Bidou Zachariasen, 1997, Proust sociologue. De la maison aristocratique au salon bourgeois. Paris, Descartes \& Cie; J. Dubois, 1997, Pour Albertine. Proust et le sens $d u$ social, Paris, Seuil (Liber). 
[...] des jeunes femmes allaient tout le jour coiffées de hauts turbans cylindriques comme aurait pu l'être une contemporaine de $\mathrm{M}^{\mathrm{me}} \mathrm{Tallien}$, par civisme, ayant des tuniques égyptiennes droites, sombres, très «guerre», sur des jupes très courtes; elles chaussaient des lanières rappelant le cothurne selon Talma, ou de hautes guêtres rappelant celles de nos chers combattants; c'est, disaient-elles, parce qu'elles n'oubliaient pas qu'elles devaient réjouir les yeux de ces combattants, qu'elles se paraient encore, non seulement de toilettes «floues», mais encore de bijoux évoquant les armées par leur thème décoratif, si même leur matière ne venait pas des armées, n'avait pas été travaillée aux armées; au lieu d'ornements égyptiens rappelant la campagne d'Égypte, c'était des bagues ou des bracelets faits avec des fragments d'obus ou des ceintures de $75,[\ldots]$ c'est encore parce qu'elles y pensaient sans cesse, disaient-elles, qu'elles en portaient, quand l'un des leurs tombaient, à peine le deuil, sous le prétexte qu'il était «mêlé de fierté», ce qui permettait un bonnet de crêpe anglais blanc [...] et même de garder ses perles, «tout en observant le tact et la correction qu'il est inutile de rappeler à des Françaises». (p. 89-90)

Un passage à lire dans tous les sens, façon Canard enchaîné, remake de La Bruyère, mais aussi de La dernière mode de Mallarmé, que Proust fait suivre d'une longue citation (ou pastiche) du discours des couturiers qui avouaient que :

«Les tristesses de l'heure [...] pourraient avoir raison des énergies féminines si nous n'avions tant de hauts exemples de courage et d'endurance à méditer. [...] Ce sera même une des plus heureuses conséquences de cette triste guerre [...] que (on attendait : la reprise des provinces perdues, le réveil du sentiment national) [...] que d'avoir obtenu de jolis résultats en fait de toilette, $[\ldots]$ avec très peu de chose $[\ldots] »($ p. 91)

Une telle manière de concevoir les rapports du front et de l'arrière a de quoi laisser pour le moins rêveur. Reste qu'elle porte à sa manière sa part de vérité, puisque aussi bien elle situe ces rapports au seul plan où ils soient concevables, celui d'une inévitable et très réelle comédie des erreurs. Une comédie des erreurs tout à la fois plaisante et touchante, cruelle et obscène, glorieuse et odieuse.

\section{La comédie des erreurs}

J'ai évoqué le double tropisme qui pour Proust était au fondement de l'ordre du discours en temps de guerre, et qui le conduit à faire une place distincte à la conversation de Saint-Loup, qu'il se garde bien de mêler à celle des salons. Et pourtant de l'un à l'autre, il s'agit aussi de ménager des relais. Proust les construira sur la voie des couturiers, mais en nous contant de tout autres histoires. À la place de la femme au foyer, d'ailleurs présente dans le récit en la personne de Gilberte trompée et délaissée, gardienne d'un Combray ravagé par la guerre, c'est une histoire d'oncle et de neveu qu'il nous racontera. Je n'entrerai pas dans 
le dédale de ce «roman passionné des homosexuels», version Saint-Loup ou version Charlus. Je me contenterai d'indiquer qu'il est un des chiffres de l'écriture proustienne de la guerre, une écriture qui procède d'une lecture à rebours du texte de la conversation française. De cette conversation française qui de part et d'autre de ses lignes de fracture, celle qui mène au front, celle qui mène à l'univers oriental et médiéval des plaisirs très particuliers du baron de Charlus, se construit sur la forclusion de l'expérience concrète de la guerre. C'est bien pourquoi, dans le roman proustien, cette conversation en vient à s'abimer, dans tous les sens du terme, dans le bordel de Jupien. Le bordel de Jupien, c'est aussi l'autre face, la face d'ombre et d'inquiétante dérision, du salon de $\mathrm{M}^{\mathrm{me}}$ Verdurin. Ce n'est d'ailleurs pas un hasard s'il est commandité par un baron, grand exclu de ce salon, superbement intelligent et fou à lier (c'est le cas de le dire), qui se fait une spécialité de relever les symétries entre ce qui se dit de part et d'autre de la frontière.

Que retenir de l'écriture proustienne de la guerre, dont j'ai essayé un peu trop rapidement de donner une idée ? Ceci tout d'abord, que malgré les apparences, il ne s'agit pas à proprement parler d'une écriture au second degré. Si Proust se montre si attentif à l'économie discursive en temps de guerre, c'est parce qu'elle est un fait de guerre à part entière, qu'elle en participe pleinement. D'où l'importance de l'appréhension et de l'intellection du dire et des manières de dire, mais aussi la nécessité pour en rendre compte d'une véritable recherche esthétique. Car rien n'est plus délicat pour l'écrivain que la quête d'une bonne distance susceptible dans le même temps de convoyer un sens de la solidarité et une perspective critique ${ }^{11}$. D'autant que tout ce qui a trait à la guerre et aux discours qu'elle engendre est pour le moins trouble et troublant. Même la souffrance et le deuil. Peut-être faudrait-il dire surtout la souffrance et le deuil ${ }^{12}$. À l'épisode du bordel de Jupien fait suite dans la Recherche la nouvelle de la mort de Saint-Loup au front, un Saint-Loup dont Proust fait un touchant éloge funèbre. Mais c'est aussi pour tracer en regard l'image plus inquiétante du chagrin de Françoise:

Elle prit immédiatement son rôle de pleureuse et commenta la mémoire du mort de lamentations, de thrènes désespérés. [....] «Pauvre dame, disait-elle, en pensant à

11. Une difficulté à laquelle les historiens se montrent désormais particulièrement sensibles. C. Ginzburg, 1998, À distance. Neuf essais sur le point de vue en histoire, traduit de l'italien par P.A. Fabre, Paris, Gallimard.

12. C. Trévisan, 2001, Les fables du deuil. La Grande Guerre : mort et écriture, Paris, PUF. 
$\mathrm{M}^{\mathrm{me}}$ de Marsantes, qu'est-ce qu'elle a dû pleurer quand elle a appris la mort de son garçon ! Si encore elle avait pu le revoir, mais il vaut peut-être mieux qu'elle n'ait pas pu, parce qu'il avait le nez coupé en deux, il était tout dévisagé.» Et les yeux de Françoise se remplissaient de larmes, mais à travers lesquelles perçait la curiosité cruelle de la paysanne. [...] Et Françoise, qui trouvait du plaisir à pleurer, ajouta encore : «il faudra bien [...] m'avertir si on cause de la mort du marquis sur le journal. » (p. 236-237)

Quand on sait, comme le sait Proust, que le chagrin d'une mère dont se repait toute une rhétorique en temps de guerre a de bonnes chances (voir Françoise) de devenir objet de délectation, on mesure mieux la responsabilité qui incombe à l'écrivain désireux de répondre à l'exigence et au défi d'écrire la guerre. Et l'on comprend sans doute que ce n'est par simple souci d'esthète, ou par gout de la novation, que Proust se refuse à simplifier son écriture et à se mettre au diapason des attentes du temps.

\section{Résumé / Abstract / Compendio}

Entre décence et obscénité : ce qui se dit de la guerre dans Le temps retrouvé

La guerre est évoquée dans Le temps retrouvé sur un mode ostensiblement déviant, à la limite de la provocation. Il y va d'un parti-pris esthétique qui remet en cause les attentes des lecteurs et leur bien-fondé. Dire la guerre consiste pour Proust à rendre sensible le lecteur aux diverses manifestations de l'ordre du discours en temps de guerre et aux évidences tout à la fois comiques et odieuses qui le sous-tendent.

Mots-clés : Proust, Le temps retrouvé, écriture provocatrice, patriotisme, civisme, conversation, comédie des erreurs.

\section{Proust's appraisal of war in Time Regained : a mixture of decency and obscenity}

World war 1914-1918 is addressed in a peculiar and almost provocative way in Proust's novel. This has to be seen as a challenging aesthetic stand. It undermines the reader's expectations, as well as the beliefs on which those expectations are grounded. Proust aims at unveiling the various expressions of the order of discourse in war time, thus bringing to our attention what we are used to consider self-evident. We are compelled to face the obvious, and to cope with its comical and unbearable oddity.

Key words : Proust, Time Regained, provocative writing, patriotism, public-spiritedness, conversation, comedy of errors. 
Entre decencia y obscenidad : lo que se dice de la guerra en Le temps retrouvé de Proust

La guerra está evocada en Le temps retrouvé bajo un modo claramente desviado, al límite de la provocación. Es una posición estética que trastorna las eperas del lector y sus ideas básicas. Para Proust consiste más bien en una sensibilización del lector a las diferentes manifestaciones del orden del discurso en tiempo de guerra y a las evidencias tal vez cómicas y odiosas que sobrentienden.

Palabras claves : Proust, Le temps retrouvé, escritura provocadora, patriotismo, civismo, conversación, comedia de los errores. 